\title{
SIMBA observations of the R Corona Australis molecular cloud ${ }^{\star} \star \star$
}

\author{
R. Chini ${ }^{1}$, K. Kämpgen ${ }^{1,2}$, B. Reipurth ${ }^{2}$, M. Albrecht ${ }^{1}$, E. Kreysa ${ }^{3}$, R. Lemke ${ }^{1}$, M. Nielbock ${ }^{4}$, \\ L. A. Reichertz ${ }^{3}$, A. Sievers ${ }^{5}$, and R. Zylka ${ }^{6}$ \\ 1 Astronomisches Institut der Ruhr-Universität Bochum, Universitätsstrasse 150, 44780 Bochum, Germany \\ 2 Institute for Astronomy, University of Hawaii, 2680 Woodlawn Drive, Honolulu, HI 96822, USA \\ 3 Max Planck Institut für Radioastronomie, Auf dem Hügel 69, 53121 Bonn, Germany \\ 4 SEST, European Southern Observatory, Alonso de Cordova 3107, Santiago, Chile \\ 5 IRAM, Avda. Divina Pastora 7, Nucleo Central, 18012 Granada, Spain \\ ${ }^{6}$ I. Physikalisches Institut, Universität zu Köln, Zülpicher Strasse 77, 50937 Köln, Germany
}

Received 10 March 2003 / Accepted 16 July 2003

\begin{abstract}
We have mapped the R Corona Australis molecular cloud at $1.2 \mathrm{~mm}$ with SIMBA on SEST and detected 25 distinct dust emission peaks. While 7 of them coincide with positions of previously known young stars, 18 are seemingly not associated with any known stellar object. We discuss the nature of individual sources and conclude that there are at least four small concentrations of young objects located along the filamentary shaped cloud. A comparison with $\mathrm{C}^{18} \mathrm{O}$ data hints at the depletion of molecules in some of the cores. Our new results yield some conflicting arguments about whether star formation proceeds from north-west to south-east in the R Cr A cloud.
\end{abstract}

Key words. ISM: dust, extinction - stars: circumstellar matter - stars: formation

\section{Introduction}

For more than a century, attention has been drawn to a high extinction region in Corona Australis where a number of variable stars are located. The R Corona Australis cloud coincides with Rossano's (1978) cloud A and is named after the highly variable Herbig Ae/Be star R Cr A (e.g. Joy 1945; Herbig 1960) which is surrounded by a bright reflection nebula that also varies with time (e.g. Graham \& Phillips 1987). A major reflection nebula surrounds the B8 star TY Cr A which is an eclipsing PMS binary (e.g. Vaz 2001) and the neighbouring A0 star HD 176386. The two bright variable stars S Cr A and VV Cr A are both T Tauri stars and visual PMS binaries (e.g. Reipurth \& Zinnecker 1993).

These young stars are associated with a large complex of dark clouds mapped in molecular and atomic transitions by, among others, Loren (1979), Cappa de Nicolau \& Pöppel (1991), Harju et al. (1993) and Yonekura et al. (1999). These data, together with large-scale extinction maps based on star

Send offprint requests to: $\mathrm{R}$. Chini,

e-mail: chini@astro.rub.de

* Based on observations collected at the European Southern Observatory, La Silla, Chile.

$\star \star$ Appendices A and B are only available in electronic form at http://www.edpsciences.org counts (e.g. Rossano 1978; Andreazza \& Vilas-Boas 1996; Cambrésy 1999), reveal a cometary shaped cloud complex oriented northwest-southeast which is about $6^{\circ}$ long with the abovementioned young stars located in the dense cometary head. This morphology suggests that star formation in the Corona Australis complex has been triggered by an external influence which possibly originates in the Upper CentaurusLupus association (Harju et al. 1993). At a distance of about 170 pc (Knude \& Høg 1998), the Cr A cloud complex is among the closest star-forming regions.

Surveys of the R Cr A cloud have revealed a population of young, visible low-mass stars, either through the presence of $\mathrm{H} \alpha$ emission (e.g. Knacke et al. 1973; Marraco \& Rydgren 1981) or as X-ray sources (e.g. Koyama et al. 1996; Neuhäuser et al. 2000). Infrared surveys have uncovered a small cluster, named the Coronet, of low-luminosity sources embedded around R Cr A (Taylor \& Storey 1984; Wilking et al. 1986; Wilking et al. 1997). More deeply embedded sources have been identified through radio continuum observations (Brown 1987; Suters et al. 1996; Harju et al. 2001) and millimetre continuum observations (Reipurth et al. 1993; Henning et al. 1994; Saraceno et al. 1996). These young stars drive various outflows which are observed either as Herbig-Haro objects or as molecular outflows (e.g. Hartigan \& Graham 1987; Levreault 1988; Graham 1993; Anderson et al. 1997). 


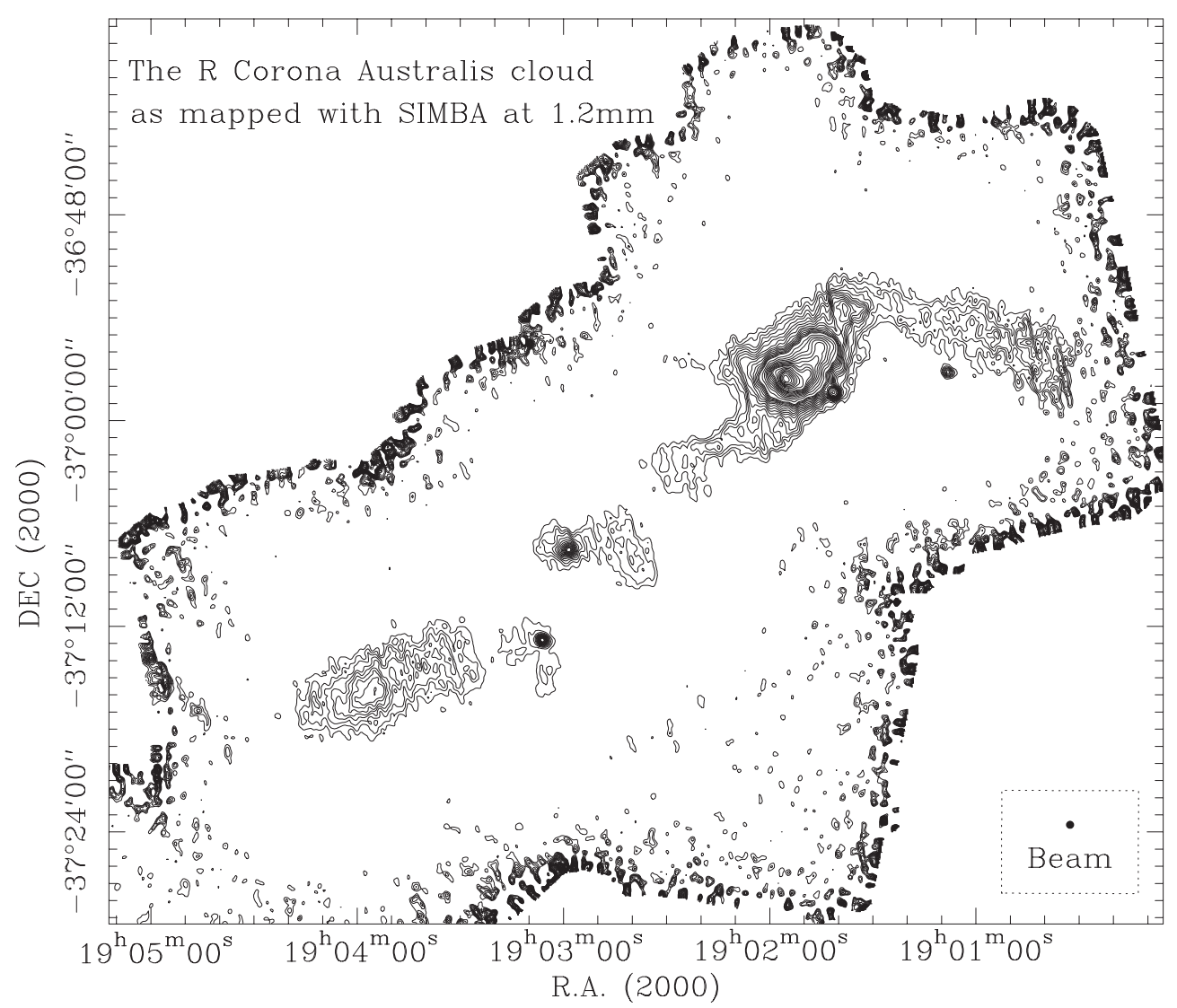

Fig. 1. The complete $1.2 \mathrm{~mm}$ mosaic of the $\mathrm{R} \mathrm{Cr}$ A dark cloud consisting of 71 individual fastscanning maps. This and all the following figures containing SIMBA data are slightly Gaussian smoothed (28" vs. a beam of $\left.24^{\prime \prime}\right)$. The smoothed beam (28") is indicated in the box to the lower right. The typical $1 \sigma \mathrm{rms}$ noise is $17 \pm 1 \mathrm{mJy} / \mathrm{beam}$; along the edges, the noise level is obviously higher. The contour lines start at $51 \mathrm{mJy} / \mathrm{beam}$ (=3 rms). Details of this mosaic are given in subsequent figures.

In this paper, we present the first deep, large-scale survey of the R Corona Australis cloud at millimetre continuum wavelengths. Our $1.2 \mathrm{~mm}$ maps reveal several small clusters of embedded, very cold objects which we interpret in terms of concentrations of newborn low-mass stars.

\section{Observations}

The $1.2 \mathrm{~mm}$ continuum observations were carried out with the 37 channel bolometer array SIMBA (SEST Imaging Bolometer Array) at the SEST (Swedish-ESO Submillimetre Telescope) on La Silla, Chile, during the commissioning periods in June, July and October 2001. We performed a mosaic that covers an area extending from north-west to southeast of roughly $2000^{\prime \prime} \times 1000^{\prime \prime}$. The mosaic was constructed by combining 71 individual fastscanning maps ( $80^{\prime \prime}$ per s) of different sizes taken at different hour angles in order to reduce scanning effects. The total integration time spent on the area was $978 \mathrm{~min}$. The residual noise in the final co-added mosaic varies by \pm 2 around $17 \mathrm{mJy} / \mathrm{beam}$ (rms) across the region due to different numbers of coverages per area. The beam size of an individual bolometer channel is $24^{\prime \prime}$ HPBW. Skydips were performed at least every three hours in order to determine the atmospheric opacity. The zenith opacity values range from 0.160 to 0.364 . The positional accuracy was determined to be $2^{\prime \prime}-3^{\prime \prime}$ by performing frequent pointing measurements. Maps of Uranus were taken for calibration purposes. A calibration accuracy of $15 \%$ was obtained. All data were reduced and analysed with MOPSI ${ }^{1}$ according to the instructions of the SIMBA Observer's Handbook (2002). A detailed description of the data reduction and the data analysis including the determination of the noise in the mosaic is presented in Appendices A and B, respectively.

\section{Results and discussion}

In the following section, we present and discuss the data from our $1.2 \mathrm{~mm}$ survey. Figure 1 shows the total mosaic of the $\mathrm{R}$ Corona Australis region as covered by SIMBA. Table 1 summarises those 25 dust emission peaks - labelled "MMS" (millimetre source) - which we consider to be bona fide detections. They were selected according to the following criteria: i) their $S / N$ ratio is larger than 9 (see Appendix B.1 for the definition of the $S / N$ ratio); ii) their extent is larger or equal to the beam size; iii) their reality was checked within individual maps in order to exclude residuals caused by the direction of fast-scanning. All entries in Table 1 are derived from the original, unsmoothed mosaic. As to be seen in the overview in Fig. 1, there are additional peaks above the $3 \sigma$

\footnotetext{
${ }^{1}$ MOPSI is a software package for infrared, millimetre and radio data reduction developed and constantly upgraded by R. Zylka.
} 
Table 1. Source positions, peak intensities, morphology, flux densities and other designations for $25 \mathrm{~mm}$ sources detected by SIMBA.

\begin{tabular}{|c|c|c|c|c|c|c|}
\hline $\begin{array}{l}\mathrm{RCr} A \\
\mathrm{MMS}\end{array}$ & \multicolumn{2}{|c|}{$(\mathrm{J} 2000)$} & $\begin{array}{c}I_{1.2} \mathrm{~mm}^{(1)} \\
{[\mathrm{mJy} / \mathrm{beam}]}\end{array}$ & $\begin{array}{c}\text { Source size }(a \times b)^{(2)} \\
{\left[{ }^{\prime \prime}\right]}\end{array}$ & $\begin{array}{l}S_{1.2 \mathrm{~mm}}^{(3)} \\
{[\mathrm{mJy}]}\end{array}$ & Other designations \\
\hline 1 & $19^{\mathrm{h}} 01^{\mathrm{m}} 08^{\mathrm{s}} .3$ & $-36^{\circ} 57^{\prime} 19^{\prime \prime}$ & $260(0)$ & $25 \times 25$ & 290 & S Cr A; ISO 116 \\
\hline 2 & $19^{\mathrm{h}} 01^{\mathrm{m}} 04^{\mathrm{s}} .2$ & $-36^{\circ} 54^{\prime} 23^{\prime \prime}$ & 140 & & & \\
\hline 3 & $19^{\mathrm{h}} 01^{\mathrm{m}} 06^{\mathrm{s}} .6$ & $-36^{\circ} 54^{\prime} 25^{\prime \prime}$ & 160 & & & \\
\hline 4 & $19^{\mathrm{h}} 01^{\mathrm{m}} 09^{\mathrm{s}} .0$ & $-36^{\circ} 54^{\prime} 18^{\prime \prime}$ & 138 & & & \\
\hline 5 & $19^{\mathrm{h}} 01^{\mathrm{m}} 14^{\mathrm{s}} .6$ & $-36^{\circ} 54^{\prime} 02^{\prime \prime}$ & 141 & & & \\
\hline 6 & $19^{\mathrm{h}} 01^{\mathrm{m}} 33^{\mathrm{s}} .2$ & $-36^{\circ} 53^{\prime} 35^{\prime \prime}$ & 268 & & & \\
\hline 7 & $19^{\mathrm{h}} 01^{\mathrm{m}} 38^{\mathrm{s}} .1$ & $-36^{\circ} 54^{\prime} 03^{\prime \prime}$ & 322 & & & \\
\hline 8 & $19^{\mathrm{h}} 01^{\mathrm{m}} 38^{\mathrm{s}} .2$ & $-36^{\circ} 53^{\prime} 36^{\prime \prime}$ & 336 & & & HD 176386 \\
\hline 9 & $19^{\mathrm{h}} 01^{\mathrm{m}} 41^{\mathrm{s}} .7$ & $-36^{\circ} 58^{\prime} 28^{\prime \prime}$ & $660(0)$ & $36 \times 32$ & 1320 & IRS 2; TS 13.1; IRAS 21 \\
\hline 10 & $19^{\mathrm{h}} 01^{\mathrm{m}} 45^{\mathrm{s}} .9$ & $-36^{\circ} 55^{\prime} 48^{\prime \prime}$ & 838 & & & \\
\hline 11 & $19^{\mathrm{h}} 01^{\mathrm{m}} 46^{\mathrm{s}} .6$ & $-36^{\circ} 56^{\prime} 30^{\prime \prime}$ & 897 & & & \\
\hline 12 & $19^{\mathrm{h}} 01^{\mathrm{m}} 49^{\mathrm{s}} .2$ & $-36^{\circ} 57^{\prime} 22^{\prime \prime}$ & 1173 & & & IRS 5; TS 2.4 \\
\hline 13 & $19^{\mathrm{h}} 01^{\mathrm{m}} 55^{\mathrm{s}} .5$ & $-36^{\circ} 57^{\prime} 38^{\prime \prime}$ & 2315 & & & confused; see text \\
\hline 14 & $19^{\mathrm{h}} 01^{\mathrm{m}} 58^{\mathrm{s}} .0$ & $-37^{\circ} 01^{\prime} 03^{\prime \prime}$ & 150 & & & \\
\hline 15 & $19^{\mathrm{h}} 02^{\mathrm{m}} 02^{\mathrm{s}} .4$ & $-37^{\circ} 01^{\prime} 34^{\prime \prime}$ & 158 & & & \\
\hline 16 & $19^{\mathrm{h}} 02^{\mathrm{m}} 05^{\mathrm{s}} .0$ & $-37^{\circ} 01^{\prime} 31^{\prime \prime}$ & 147 & & & \\
\hline 17 & $19^{\mathrm{h}} 02^{\mathrm{m}} 04^{\mathrm{s}} .8$ & $-37^{\circ} 00^{\prime} 03^{\prime \prime}$ & 180 & & & \\
\hline 18 & $19^{\mathrm{h}} 02^{\mathrm{m}} 08^{\mathrm{s}} .6$ & $-37^{\circ} 00^{\prime} 41^{\prime \prime}$ & 170 & & & \\
\hline 19 & $19^{\mathrm{h}} 02^{\mathrm{m}} 12^{\mathrm{s}} .4$ & $-36^{\circ} 57^{\prime} 17^{\prime \prime}$ & 146 & & & \\
\hline 20 & $19^{\mathrm{h}} 02^{\mathrm{m}} 15^{\mathrm{s}} .3$ & $-37^{\circ} 01^{\prime} 37^{\prime \prime}$ & 160 & & & \\
\hline 21 & $19^{\mathrm{h}} 02^{\mathrm{m}} 17^{\mathrm{s}} .8$ & $-37^{\circ} 01^{\prime} 34^{\prime \prime}$ & 148 & & & \\
\hline 22 & $19^{\mathrm{h}} 02^{\mathrm{m}} 40^{\mathrm{s}} .7$ & $-37^{\circ} 08^{\prime} 11^{\prime \prime}$ & 146 & & & \\
\hline 23 & $19^{\mathrm{h}} 02^{\mathrm{m}} 59^{\mathrm{s}} .0$ & $-37^{\circ} 07^{\prime} 32^{\prime \prime}$ & $472(126)$ & $36 \times 31$ & 672 & $\begin{array}{c}\text { IRAS 18595-3712; IRAS 32c; } \\
\text { ISO-CrA } 182\end{array}$ \\
\hline 24 & $19^{\mathrm{h}} 03^{\mathrm{m}} 06^{\mathrm{s}} .9$ & $-37^{\circ} 12^{\prime} 47^{\prime \prime}$ & $543(42)$ & $28 \times 24$ & 584 & VV Cr A; IRAS 18597-3717 \\
\hline 25 & $19^{\mathrm{h}} 03^{\mathrm{m}} 56^{\mathrm{s}} .1$ & $-37^{\circ} 15^{\prime} 32^{\prime \prime}$ & 250 & & & \\
\hline
\end{tabular}

(1) Peak intensity measured above the zero level; the beam size is $24^{\prime \prime}$. The figures in brackets denote the level of the local background intensity.

(2) FWHM of major and minor axis as derived from a two-dimensional Gaussian fit where possible.

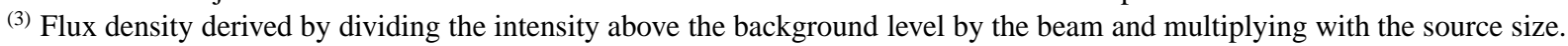

level which, however, might be residuals from the deconvolution (see Appendix A.1.5) and thus deserve further integration time in order to be classified as true detections. In general, the classification of certain contours as a dust emission peak is not always unique due to lacking spatial resolution. Thus, it might turn out that regions which are treated as a ridge in the present paper may be resolved into distinct peaks by higher resolution data and vice versa.

For reasons of clarity, we divide the cloud into a northwestern and a south-eastern part. We will discuss the $25 \mathrm{~mm}$ sources in Sect.3.1 and Sect. 3.2 taking into account all available optical, infrared and radio information. A comparison between dust and molecular emission is made in Sect. 3.3. A description of the nature of star formation within the $\mathrm{R} \mathrm{Cr}$ A cloud concludes this section.

\subsection{Sources in the north-western part of the cloud}

The north-western portion of the cloud contains more than $80 \%$ of all the detected dust emission peaks in the $\mathrm{R} \mathrm{Cr} \mathrm{A}$ region. Figure 2 depicts an enlargement of the corresponding area showing 21 bona fide detections at $1.2 \mathrm{~mm}$. Further peaks located west of MMS 2 can be seen in Fig. 1 which, however, are not labelled due to the insufficient observational coverage of this region. Altogether 17 out of the 21 dust emission peaks in the north-western region have been detected at $1.2 \mathrm{~mm}$ for the first time.

\subsubsection{MMS 1}

The strong continuum source MMS 1 coincides within positional errors with the bright T Tauri star S Cr A. This is one of the original T Tauri stars discovered by Joy (1945) and is a $1^{\prime \prime}$ visual binary which has been studied extensively (e.g. Appenzeller \& Wolf 1977; Wilking et al. 1985, 1992). Although located in a tenuous medium outside the main cloud core, it has strong outflow activity as evidenced by its association with HH objects (e.g. Strom et al. 1986). Olofsson et al. (1999) reported strong emission and excess at mid-infrared wavelengths. S Cr A was originally found to be a strong millimetre continuum source by Reipurth et al. (1993).

\subsubsection{The MMS $2-5$ group}

The weakest millimetre sources are located in a ridge of dust which stretches over at least $12^{\prime}$ to the north-west of the main core. They are not associated with any known young stellar objects. A few mid-infrared sources were detected by Olofsson et al. (1999) but, since none of them shows any flux at $14.3 \mu \mathrm{m}$, they are likely to be stars not associated with the molecular 


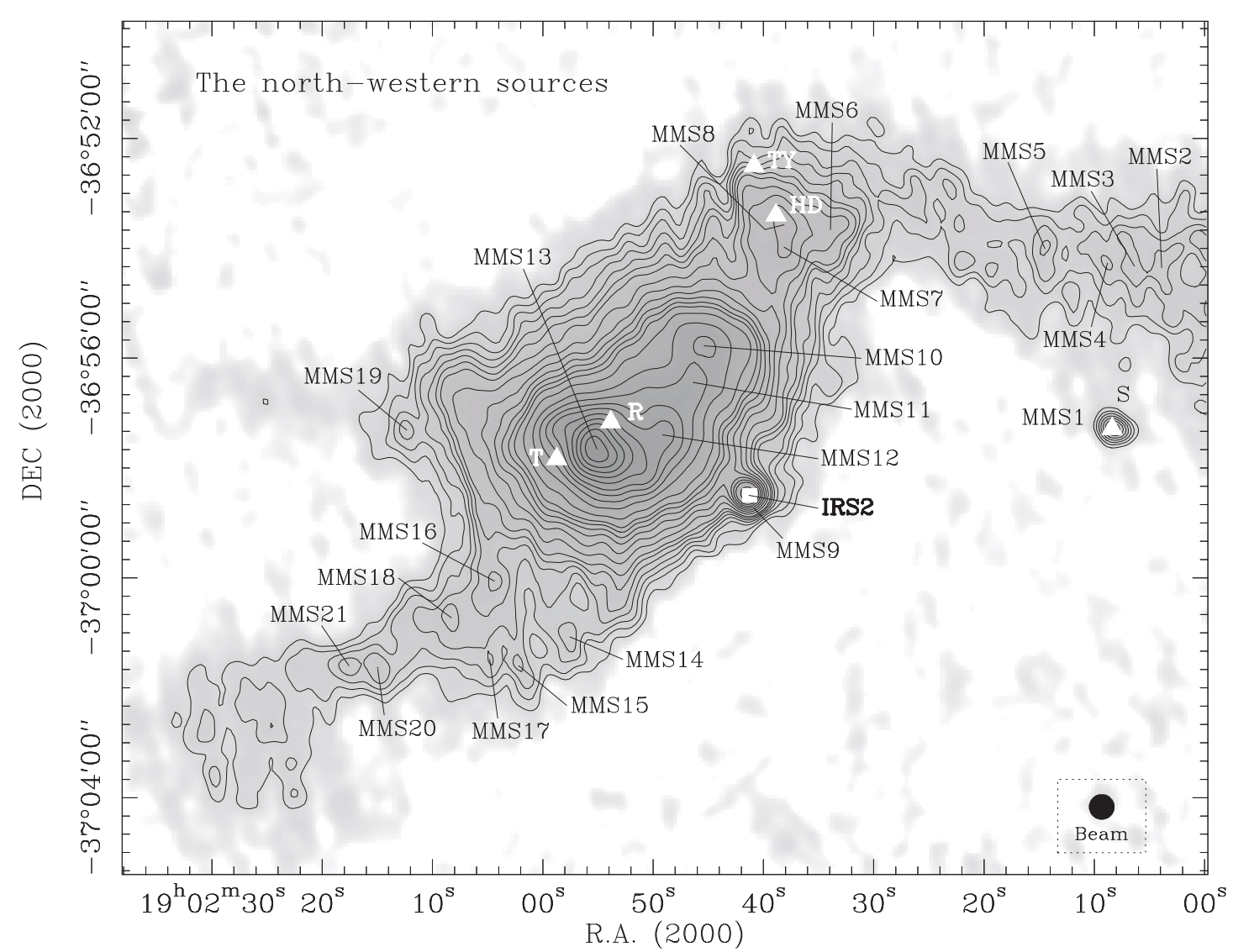

Fig. 2. The north-western part of the cloud. The millimetre sources are generally labelled according to their right ascension with the exception of Nos. 1, 2 and 3 where we deviated from this scheme in order to maintain a sequence within physically related structures. Triangles denote the positions of the known visible stars S Cr A, HD 176386, TY Cr A, R Cr A and T Cr A. The near-infrared source IRS 2 (Taylor \& Storey 1984) is marked with a square. The contours are 72 to 252 in steps of 30, 305 to 580 in steps of 55,690 to 1450 in steps of 190 , and 1720 to 2800 in steps of 270 . The units are in $\mathrm{mJy} /$ beam. Note: due to the fact that the data are Gaussian smoothed to $28^{\prime \prime}$ and the large dynamical range of the peak intensities, it is not possible to improve the visibility of the peaks without overloading the figure with too many contours.

cloud. Therefore, the nature of these millimetre sources is hard to constrain. Due to their location west of $\mathrm{R} \mathrm{Cr} A$, the site of vigorous star formation, and assuming that the picture of triggered star formation from northwest to southeast holds for the entire cloud, it is possible that they simply represent density enhancements of the cloud. In order to classify them as possible protostellar condensations, further observations are required.

\subsubsection{The MMS 6-8 group}

Located in the immediate vicinity of the two Herbig Ae/Be stars TY CrA and HD 176386, which dominate the visual appearance of this part of the R CrA cloud with their extended reflection nebulae NGC 6726/7, we find a little cluster of millimetre sources. TY CrA is clearly located outside the peak contour of this group and is probably not a significant contributor to the dust emission (see Fig. 2). This fact is supported by the non-detection at the same wavelength, but using ON-OFF measurements, by Henning et al. (1994). HD 176386 is located very close to the peak of MMS 8 and is seemingly associated with the emission in this area. Thus, it is possible that HD 176386 is the heating source of this little cluster. Although there are a few near-infrared
(Wilking et al. 1986, 1992, 1997) and far-infrared sources (Wilking et al. 1985, 1992) in the neighbourhood of the millimetre cluster, none of them coincides with any of the peaks in our map. We conclude that MMS 6 and MMS 7 represent embedded and presumably very young objects.

\subsubsection{MMS 9}

This bright millimetre continuum source coincides with the bright infrared Coronet member IRS 2 (Taylor \& Storey 1984; Wilking et al. 1997), see Fig. 2. Far-infrared data suggest that it is a Class I object (Wilking et al. 1985, 1992).

\subsubsection{The MMS 10-13 group}

In the general region of $\mathrm{R}$ and $\mathrm{TCr} \mathrm{A}$, we find a compact group of four millimetre sources. The brightest among them - MMS 13 - was already detected by Henning et al. (1994).

MMS 10 and MMS 11 are the northernmost bright sources in this chain of objects and clearly show an elongated morphology. There is a detection of an ammonia core at the south-eastern edge of MMS 10 (Jijina et al. 1999) but neither MMS 10 nor MMS 11 are coincident with any known near- or 


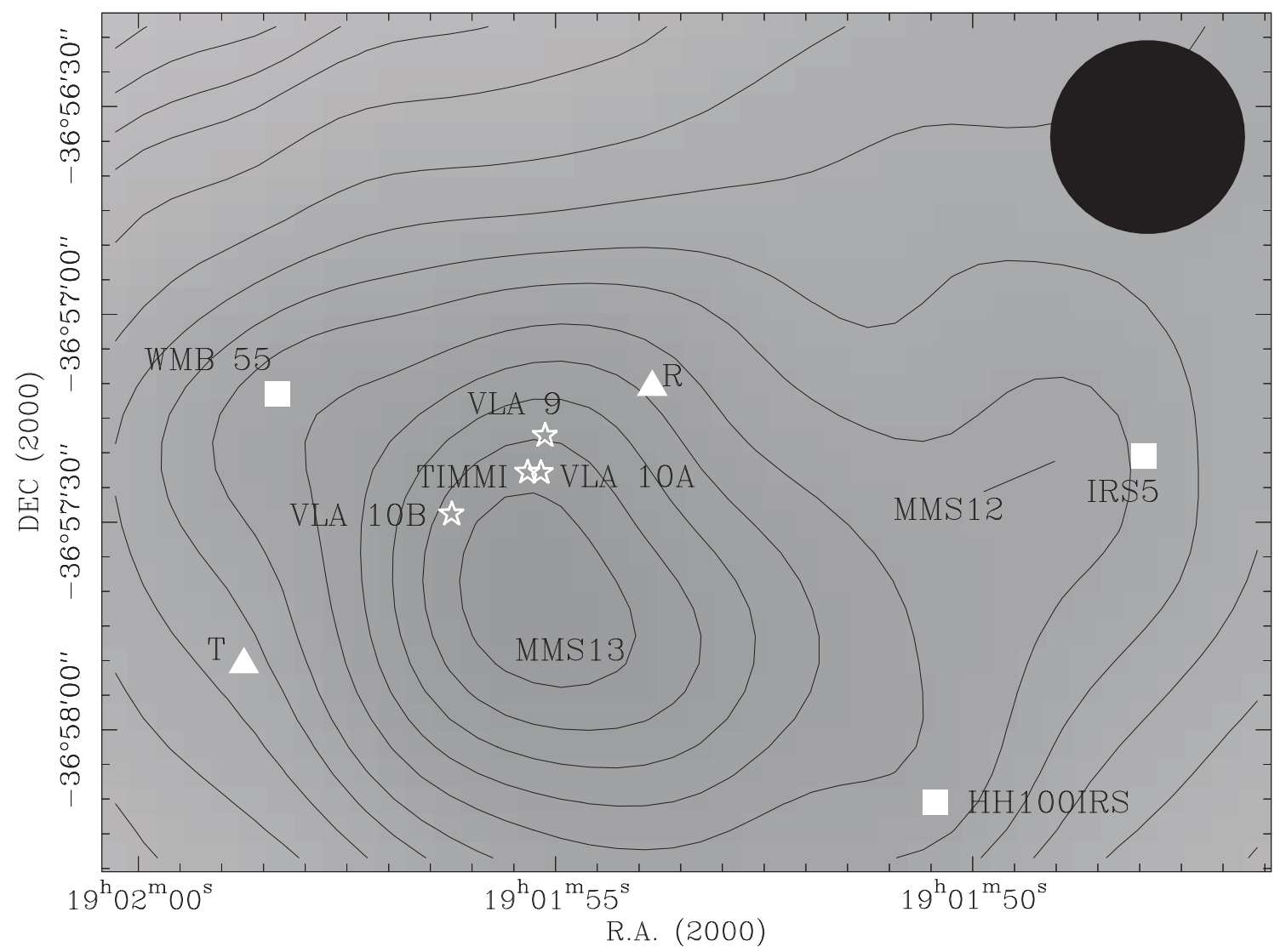

Fig. 3. Closeup view of the region around MMS 13. Triangles denote the positions of the known visible stars R Cr A and T Cr A. Near-infrared sources are marked as squares: IRS 5 (Taylor \& Storey 1984), HH 100 IRS (Elias 1980) and WMB 55 (nomenclature taken from Gezari et al. 1999, data by Wilking et al. 1997). Open stars denote the positions the three VLA sources 9, $10 \mathrm{~A}$ and $10 \mathrm{~B}$ (Brown 1987) as well as a 10 $\mu \mathrm{m}$ source labelled TIMMI (Wilking et al. 1997). The contours are 415 to 580 in steps of 55, 690 to 1450 in steps of 190 and 1720 to 2800 in steps of 270 with units in $\mathrm{mJy} / \mathrm{beam}$. The data are smoothed to $28^{\prime \prime}$ and the beam size is indicated in the upper right-hand corner.

far-infrared nor radio continuum emission sources. We assume that they represent deeply embedded young objects.

MMS 12 is identified with the bright source IRS 5 of Taylor \& Storey (1984) which is surrounded by a large infrared reflection nebula (Castelaz \& Hackwell 1987). The source underwent a major outburst in the radio continuum while observed by Suters et al. (1996). Infrared images show that the object is a 0.6" binary system (Chen \& Graham 1993). Saraceno et al. (1996) also did millimetre continuum measurements of this source and acknowledged its extended nature.

MMS 13 is by far the brightest millimetre source in our survey. Henning et al. (1994) concluded that it is not associated with any of the optically visible sources $\mathrm{R}$ and $\mathrm{TCr} \mathrm{A}$; this is confirmed by our more detailed map seen in Fig. 3. Instead, they suggested that MMS 13 is related to IRS 7 of Taylor \& Storey (1984). We have carefully examined all available photometric and astrometric observations of objects in this region and plot all known sources in Fig. 3). IRS 7 drew attention when Brown (1987) found that it is located between two radio continuum sources, suggesting that it could be the source of a bipolar radio flow. Wilking et al. (1997) found that IRS 7 is a nebulous structure adjacent to a $10 \mu \mathrm{m}$ source and, therefore, probably just represents scattered light. In Fig. 3, we plot the positions of the two radio continuum sources (labelled VLA 10A and 10B), the $10 \mu \mathrm{m}$ source (labelled TIMMI) and another nearby radio continuum source (labelled VLA 9) from Brown (1987). All positions are expected to be very accurate (the $10 \mu \mathrm{m}$ source position was measured relative to $\mathrm{R} \mathrm{Cr} \mathrm{A}$ ), and it follows that MMS 13 is neither coincident with the TIMMI/IRS 7 source nor with any of the three known VLA sources in the region. We conclude that MMS 13 is a deeply embedded source, probably of Class 0 , which has no known counterparts at other wavelengths. Given its large flux, which corresponds to $5 M_{\odot}$ for an average dust temperature $T_{\mathrm{d}}$ of $20 \mathrm{~K}$, and its proximity to R Cr A and T Cr A, we speculate that this source - if it is a single object - may be a proto-Herbig-Ae/Be star.

The little dent south-west of MMS 13 coincides with HH 100 IRS which is a well-studied, bright near-infrared source associated with the Herbig-Haro object HH 100 (Strom et al. 1974). The source is likely to also drive the larger $\mathrm{HH}$ flow that includes HH 99 and HH 101 (e.g. Hartigan \& Graham 1987). HH 100 IRS was previously observed at millimetre continuum wavelength by Reipurth et al. (1993), Henning et al. (1994) and Saraceno et al. (1996) and was detected at $6 \mathrm{~cm}$ by Brown (1987). It is a Class I object (Wilking et al. 1992).

There is another little dent to the east of MMS 13 which coincides with a near-infrared source observed by 
Wilking et al. (1997) and is listed without a number in their Table 1. We use the designation WMB 55 as employed for this star in the Catalogue of Infrared Observations, 5. Edition by Gezari et al. ${ }^{2}$. Wilking et al. (1997) detected the star at $H$ and $K$, but not at $J$, suggesting that it may be a highly extincted object. We assume that the dent is identical with this infrared source and represents a young star perhaps similar to HH 100 IRS and IRS 5.

\subsubsection{The MMS 14-21 group}

These five sources are relatively faint and their nature is unclear. They may represent embedded sources but could possibly also be density enhancements in the cloud indicating sites of future star formation. Further observations at submillimetre wavelengths are required to settle this question.

\subsection{Sources in the south-eastern part of the cloud}

Within the south-eastern part of the cloud (see Fig. 4), we detected only $4 \mathrm{~mm}$ sources with 2 of them coinciding with previously known objects. Due to insufficient coverage, the mosaic exhibits strong scanning effects in the vicinity of MMS 25, forcing us to refrain from labelling any further possible sources in that region.

\subsubsection{MMS 22}

Located in a small extended area of millimetre continuum emission, the faintest of our detected sources in the southeastern region does not have any known stellar counterparts at other wavelengths. In order to answer the question whether it represents an embedded source or just a dense core with possible future star formation activity, further investigations, especially at submillimetre wavelengths, are needed.

\subsubsection{MMS 23}

This is a bright millimetre source that coincides with the IRAS source 18595-3712 which is located towards one of the most obscured parts of the R Cr A cloud. Wilking et al. (1992) detected a small group of near-infrared sources around the IRAS position of which their source IRAS 32c is virtually coincident with our MMS 23. Olofsson et al. (1999) used ISOCAM observations to reveal two mid-infrared excess sources of which ISO-Cr A 182 is only $5^{\prime \prime}$ away from the peak of MMS 23. It is likely that IRAS 18959-3712, IRAS 32c and ISO-Cr A 182 are detections of the same object. The energy distribution is that of a Class I object.

\subsubsection{MMS 24}

Our millimetre source precisely coincides with the young visible star VV Cr A. This variable T Tauri star is well-studied at optical, infrared and millimetre wavelengths (e.g. Appenzeller et al. 1986; Graham 1992; Wilking et al. 1985, 1992;

\footnotetext{
2 Available at http://vizier.u-strasbg.fr/viz-bin/ Cat?II/225
}

Henning et al. 1994; Chelli et al. 1995; Koresko et al. 1997; Prato \& Simon 1997) and is found to be a visual binary with a late-K primary and a luminous infrared companion.

\subsubsection{MMS 25}

Located in a dense cloud core situated in the south-eastern extremity of the R CrA cloud, we find a large complex of extended millimetre emission which is located near the centre of a neutral atomic hydrogen ring with a diameter of $40^{\prime}$ (Llewellyn et al. 1981). This ring is thought to represent the outer boundary of a massive $\mathrm{HI}$ to $\mathrm{H}_{2}$ conversion region. No known young stellar objects are associated with this region, but Harju et al. (1993) estimated it to be gravitationally bound. This is contradicted by Kontinen et al. (2000) who see indications for a rather low star formation capacity in an advanced chemical stage in their data consisting of various molecular line transitions. Nevertheless, further data are needed in order to establish whether MMS 25 is a pre-stellar core in the process of becoming one or more stars or whether it is a quiescent core.

\subsection{Gas and dust emission}

In order to explore the nature of the new millimetre sources in more detail, we compare the molecular line emission from the $\mathrm{R} \mathrm{Cr}$ A cloud with our results from SIMBA. As shown in Fig. 5, the $\mathrm{C}^{18} \mathrm{O}$ emission mapped by Harju et al. (1993) follows the morphology of the optical extinction very tightly and exhibits several dense cores labelled A $1, \mathrm{~A} 2$, and $\mathrm{NE} / \mathrm{SW}$ jaw in the north-western part, A 3 and B in the central part and C and D in the south-eastern part of the cloud. Table 2 gives a detailed comparison between these $\mathrm{C}^{18} \mathrm{O}$ features and the $1.2 \mathrm{~mm}$ emission. All of the labelled $\mathrm{C}^{18} \mathrm{O}$ cores have more or less equally extended counterparts in the $1.2 \mathrm{~mm}$ mosaic. There is an additional millimeter peak at the position of S Cr A. Furthermore, a large region of extended dust emission west of $\mathrm{R} \mathrm{Cr} \mathrm{A}$ also shows in the $\mathrm{C}^{18} \mathrm{O}$ map but is not labelled there.

The $1.2 \mathrm{~mm}$ flux densities were determined by matching circular or elliptical apertures to the individual sources. The integration within different apertures for each region yielded deviations below $3 \%$ which supports the reliability of the method. Likewise, the results were confirmed by integrating over the area outlined by the $3 \mathrm{rms}$ contours. The differences between the two methods are within 15\% with the exception of the SW jaw where the value derived with the aperture method is by a factor of two higher. This fact confirms the extended but very faint nature of the millimetre emission in this region.

Dust masses were derived from the optically thin $1.2 \mathrm{~mm}$ emission according to $M_{\text {dust }}=S_{v} D^{2} / \kappa_{v, \mathrm{~d}} B_{v}\left(T_{\mathrm{d}}\right)$, where $S_{v}$ denotes the observed dust emission, $\kappa_{v}$ is the mass absorption cross section per gram of dust and $B_{v}$ is the Planck function. Owing to the lack of data at far-infrared wavelengths for most of the sources, an average dust temperature $T_{\mathrm{d}}=20 \mathrm{~K}$ was assumed. A temperature scatter of $\pm 5 \mathrm{~K}$ would imply a corresponding mass scatter of about $-25 \%$ and $+50 \%$, respectively.

The gas masses were calculated using $\kappa_{v, \mathrm{~d}}=0.37 \mathrm{~cm}^{2} \mathrm{~g}^{-1}$ and a gas-to-dust ratio of 150 (see e.g. Krügel et al. 1998). 


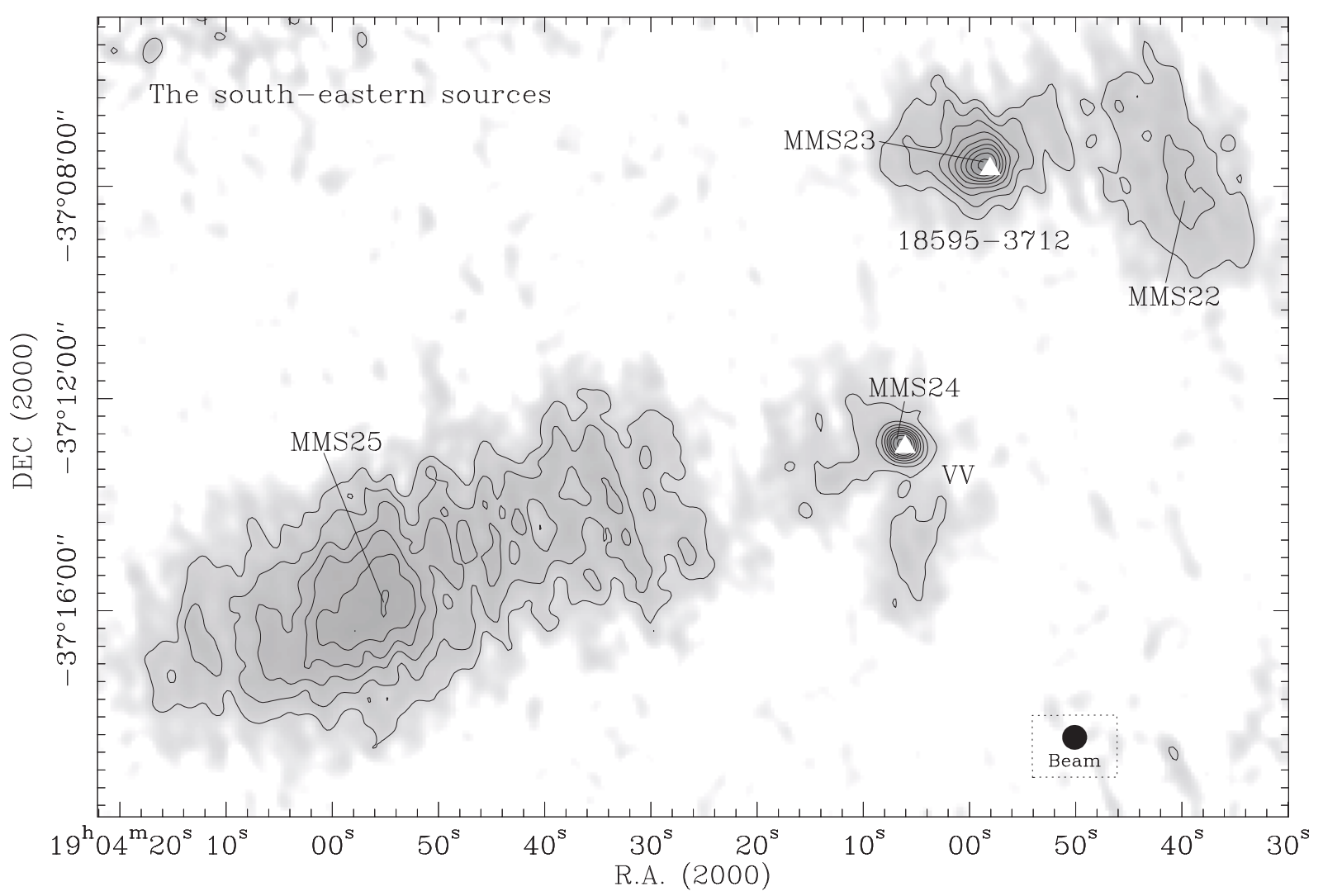

Fig. 4. Sources in the south-eastern cloud core. The millimetre sources are labelled according to their right ascension; the positions of the the IRAS source 18595-3712 and the visible star VV Cr A are marked by triangles. The contours are 70 to 230 in steps of 40 and 290 to 410 in steps of 60 . The units are in mJy/beam and the data are Gaussian smoothed to $28^{\prime \prime}$.

While these values are appropriate for the diffuse ISM, $\kappa_{v}$ may increase by up to a factor of 10 in dense regions and/or protostellar environments (e.g. Ossenkopf \& Henning 1994); such dust properties would decrease the inferred masses correspondingly. In order to allow an easy comparison between the dust and the $\mathrm{C}^{18} \mathrm{O}$ results, the gas masses derived from the $\mathrm{C}^{18} \mathrm{O}$ emission were recalculated for a distance of $170 \mathrm{pc}$ (Harju et al. 1993 used a distance of $130 \mathrm{pc}$ ). The same was done for the radii listed in Table 2.

Apart from one region, namely A 3 , the masses derived from the dust measurements exceed those from $\mathrm{C}^{18} \mathrm{O}$ systematically. Due to the effect of optical depth in the line observations on one side and due to the dust properties on the other side, the results from $\mathrm{CO}$ and dust might reflect the lower and upper limits of the mass range, respectively. However, taking into account the large uncertainties inherent in both methods, the results for most regions can as well be regarded as comparable. Only the values for A 1 and A 2 deviate by factors of 7 and 22 , respectively, which comes close to the uncertainty limits of the methods. This disagreement might indicate a depletion of molecules which is a well-known phenomenon and occurs in cold and extremely dense regions.

\subsection{Star formation in the $\mathrm{R} C r$ A cloud}

Due to the difficulties of assigning a total flux to the individual dust condensations - taking into account their location within larger extended complexes - it is impossible to derive proper masses. Using the face values of the peak flux densities from Table 1, the apparent mass distribution shows a high abundance of low-mass objects; only very few clumps have medium masses while MMS 13 is at the high mass end.

Another result of this $1.2 \mathrm{~mm}$ survey is that extended dust emission also occurs in regions harbouring fairly evolved YSOs, e.g. VVCr A, which is a visible T Tauri star. While the strong, pointlike millimetre emission probably originates from an unresolved protoplanetary disk/envelope structure, the faint extended emission represents the remnant dust lane out of which the star formed. This dust lane seems to contain further density enhancements which could become the sites of future star formation. A similar scenario appears to apply to the most massive dust cores of the R Cr A cloud, namely MMS 10-13. In their neighbourhood, many objects of different evolutionary stages and thus of different ages are crowded in a comparatively small area. We believe that the four millimetre sources are among the youngest objects or groups of objects within the cluster of YSOs.

With regard to the proposed triggering of star formation in the R Cr A molecular cloud, the new results provide two slightly conflicting arguments: on the one hand, the existence of a cloud core without any signs of ongoing star formation, namely MMS 25, in the south-easternmost part of the cloud speaks in favour of a scenario where the trigger is located west of the cloud. On the other hand, the question seems justified 


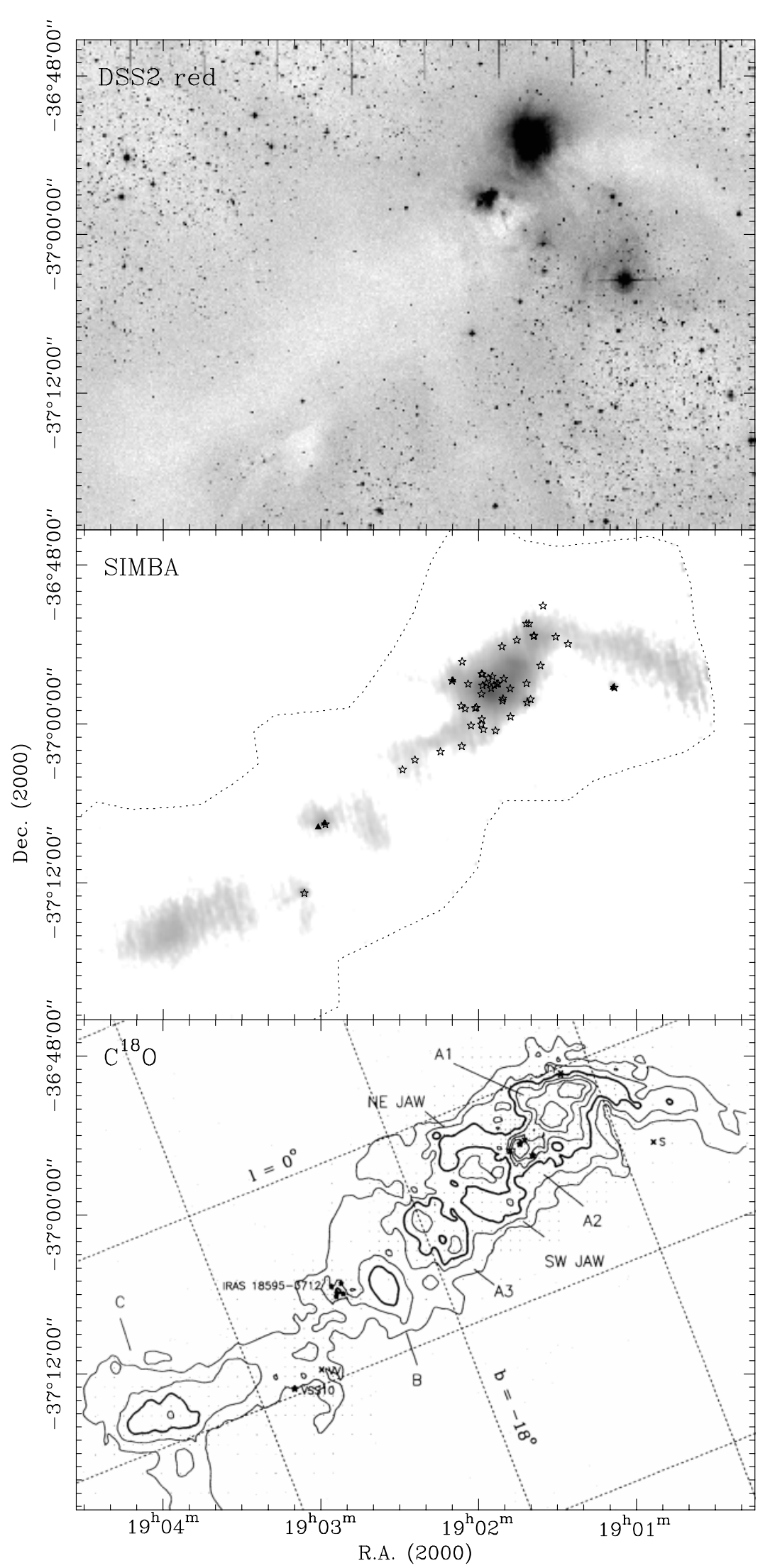

Fig. 5. The R Cr A cloud at different wavelengths. Top: Red image from the DSS2 Middle: Grey scale image of our 1.2 mm SIMBA mosaic; the dashed line outlines the extension of the SIMBA mosaic; open stars show the distribution of YSOs as observed or listed by Wilking et al. (1992, 1997) and confirmed by us using $(J-H)-(H-K)$-diagramms; solid triangles denote ISOCAM sources with mid-infrared excess (Olofsson et al. 1999) Bottom: $\mathrm{C}^{18} \mathrm{O}$ contour lines from 1 to 3.5 in steps of $0.5 \mathrm{~K} \mathrm{~km} \mathrm{~s}^{-1}$ taken from Harju et al. (1993). 
Table 2. Comparison between dust and $\mathrm{C}^{18} \mathrm{O}$ emission (Harju et al. 1993).

\begin{tabular}{|c|c|c|c|c|c|c|c|c|c|c|}
\hline \multicolumn{2}{|c|}{$\overline{\mathrm{RCrA}}$} & \multicolumn{2}{|c|}{$\begin{array}{c}\text { Position }^{(1)} \\
(\mathrm{J} 2000)\end{array}$} & \multicolumn{2}{|c|}{$\begin{array}{c}\text { Offset }^{(2)} \\
{\left[{ }^{\prime \prime}\right]}\end{array}$} & \multicolumn{2}{|c|}{$\begin{array}{l}r^{(3)} \\
{[\mathrm{pc}]}\end{array}$} & \multirow{2}{*}{$\begin{array}{c}S_{1.2 \mathrm{~mm}}{ }^{(4)} \\
{[\mathrm{Jy}]} \\
1.2 \mathrm{~mm}\end{array}$} & \multicolumn{2}{|c|}{$\begin{array}{l}M_{\text {gas }} \\
{\left[M_{\odot}\right]}\end{array}$} \\
\hline MMS & $\mathrm{C}^{18} \mathrm{O}$ & RA & Dec & RA & Dec & $\mathrm{C}^{18} \mathrm{O}$ & $1.2 \mathrm{~mm}$ & & $\mathrm{C}^{18} \mathrm{O}^{(5)}$ & $1.2 \mathrm{~mm}^{(6)}$ \\
\hline 1 & & $19^{\mathrm{h}} 01^{\mathrm{m}} 08^{\mathrm{s}} \cdot 3$ & $-36^{\circ} 57^{\prime} 19^{\prime \prime}$ & -564 & 7 & & 0.02 & 0.3 & & $\overline{0.6}$ \\
\hline $2-5$ & & $19^{\mathrm{h}} 00^{\mathrm{m}} 52^{\mathrm{s}} .2$ & $-36^{\circ} 55^{\prime} 36^{\prime \prime}$ & -757 & 111 & & 0.08 & 17.0 & & 34.5 \\
\hline $6-8,10,11$ & A1 & $19^{\mathrm{h}} 01^{\mathrm{m}} 42^{\mathrm{s}} .0$ & $-36^{\circ} 54^{\prime} 39^{\prime \prime}$ & -160 & 165 & 0.08 & 0.09 & 37.0 & 10 & 75.1 \\
\hline $12-13$ & $\mathrm{~A} 2$ & $19^{\mathrm{h}} 01^{\mathrm{m}} 53^{\mathrm{s}} .7$ & $-36^{\circ} 57^{\prime} 38^{\prime \prime}$ & -20 & -15 & 0.04 & 0.07 & 37.0 & 3 & 75.1 \\
\hline $6-21$ & A & $19^{\mathrm{h}} 01^{\mathrm{m}} 58^{\mathrm{s}} .8$ & $-36^{\circ} 58^{\prime} 07^{\prime \prime}$ & 40 & -45 & 0.21 & 0.21 & 99.6 & 80 & 202.0 \\
\hline $14-18$ & SW jaw & $19^{\mathrm{h}} 02^{\mathrm{m}} 04^{\mathrm{s}} .7$ & $-37^{\circ} 01^{\prime} 24^{\prime \prime}$ & 110 & -242 & 0.08 & 0.08 & 7.7 & 5 & 15.6 \\
\hline 19 & NE jaw & $19^{\mathrm{h}} 02^{\mathrm{m}} 15^{\mathrm{s}} .8$ & $-36^{\circ} 57^{\prime} 21^{\prime \prime}$ & 245 & 0 & 0.07 & 0.07 & 3.8 & 5 & 7.7 \\
\hline $20-21$ & A3 & $19^{\mathrm{h}} 02^{\mathrm{m}} 24^{\mathrm{s}} .3$ & $-37^{\circ} 03^{\prime} 06^{\prime \prime}$ & 345 & -345 & 0.10 & 0.07 & 4.5 & 10 & 9.1 \\
\hline $22-23$ & B & $19^{\mathrm{h}} 02^{\mathrm{m}} 46^{\mathrm{s}} .9$ & $-37^{\circ} 07^{\prime} 24^{\prime \prime}$ & 615 & -605 & 0.12 & 0.10 & 7.9 & 10 & 16.0 \\
\hline 24 & D & $19^{\mathrm{h}} 03^{\mathrm{m}} 05^{\mathrm{s}} .3$ & $-37^{\circ} 14^{\prime} 43^{\prime \prime}$ & 835 & -1045 & 0.12 & 0.05 & 2.2 & 3 & 4.5 \\
\hline 25 & $\mathrm{C}$ & $19^{\mathrm{h}} 03^{\mathrm{m}} 55^{\mathrm{s}} .0$ & $-37^{\circ} 15^{\prime} 26^{\prime \prime}$ & 1425 & -1090 & 0.16 & 0.13 & 24.7 & 19 & 50.1 \\
\hline
\end{tabular}

(1) Central position of the apertures which were used to derive the flux densities. Positions correspond to the positions of the clump centre of mass of $\mathrm{C}^{18} \mathrm{O}$ in most cases (exception: MMS 25).

(2) Offsets of the central position of the apertures relative to the position of IRS $7\left(19^{\mathrm{h}} 01^{\mathrm{m}} 55^{\mathrm{s}} .4,-36^{\circ} 57^{\prime} 23^{\prime \prime}\right.$ (J2000)) for comparison with the $\mathrm{C}^{18} \mathrm{O}$ offsets.

(3) Clump half-power radius (geometric mean) calculated for a distance of $170 \mathrm{pc}$. It was derived within an aperture that contained half of the total flux density.

(4) Flux density derived by integration (details see Sect. 3.3).

(5) Masses derived by Harju et al. (1993) but recalculated for the adopted distance of $170 \mathrm{pc}$.

(6) Gas masses of the clumps calculated according to the procedure described in Sect. 3.3.

why the dust ridge north-west of $\mathrm{R} \mathrm{Cr}$ A also lacks those signs. However, an attempt to answer this question has to be postponed until further observational and theoretical arguments are available.

\section{Conclusions}

The present study has revealed 25 sources at $1.2 \mathrm{~mm}$. We suggest that these sources reflect the youngest population of an ongoing star formation process in the $\mathrm{R} \mathrm{Cr}$ A region. Mass estimates of the millimetre clumps indicate that mainly low-mass objects are created. The fact that cold millimetre clumps are found in both the north-western and the south-eastern part of the cloud argues against a trigger scenario that runs over the cloud from the north-west to the south-east. For many sources the spatial resolution of SIMBA is not sufficient to resolve the structure or the multiplicity of dust clumps; the region has to await ALMA before its millimetre population can be analyzed in more detail.

Acknowledgements. We wish to thank all our colleagues that helped with the development of SIMBA and brought it into a successful operation; in particular we want to mention W. Esch, H.-P. Gemünd and M. Anciaux. We also express our thanks to the SEST team for its excellent support during the installation and the first observations with the new instrument. Likewise, we acknowledge the good collaboration with ESO during the construction and commissioning of SIMBA. It is a pleasure for R.C. and K.K. to acknowledge the hospitality of the Institute for Astronomy in Honolulu, Hawai' $i$, where most of the reduction and analysis work for the present paper were performed. Finally, we thank our referee Dr. M. Juvela who helped to clarify and to improve the paper by numerous substantial comments.

This research has made use of the SIMBAD database, operated at CDS, Strasbourg, France.

\section{References}

Anderson, I. M., Harju, J., Knee, L. B. G., \& Haikala, L. K. 1997, A\&A, 321, 575

Andreazza, C. M., \& Vilas-Boas, J. W. S. 1996, A\&AS, 116, 21

Appenzeller, I., Jetter, R., \& Jankovics, I. 1986, A\&AS, 64, 65

Appenzeller, I., \& Wolf, B. 1977, A\&A, 54, 713

Brown, A. 1987, ApJ, 322, L31

Cambrésy, L. 1999, A\&A, 345, 965

Cappa de Nicolau, C. E., \& Pöppel, W. G. L. 1991, A\&AS, 88, 615

Castelaz, M. W., \& Hackwell, J. A. 1987, ApJ, 314, 317

Chelli, A., Cruz-Gonzalez, I., \& Reipurth, B. 1995, A\&AS, 114, 135

Chen, W. P., \& Graham, J. A. 1993, ApJ, 409, 319

Elias, J. H. 1980, ApJ, 241, 728

Gezari, D. Y., Pitts, P. S., \& Schmitz, M. 1999, Catalog of Infrared Observations, Edition 5

Graham, J. A. 1992, PASP, 104, 479

Graham, J. A. 1993, PASP, 105, 561

Graham, J. A., \& Phillips, A. C. 1987, PASP, 99, 91

Harju, J., Haikala, L. K., Mattila, K., et al. 1993, A\&A, 278, 569

Harju, J., Higdon, J. L., Lehtinen, K., \& Juvela, M. 2001, in Science with the Atacama Large Millimeter Array, ed. A. Wootten, ASP, 125

Hartigan, P., \& Graham, J. A. 1987, AJ, 93, 913

Henning, Th., Launhardt, R., Steinacker, J., \& Thamm, E. 1994, A\&A, 291, 546

Herbig, G. H. 1960, ApJS, 4, 337

Joy, A. H. 1945, ApJ, 102, 168

Knacke, R. F., Strom, K. M., Strom, S. E., Young, E., \& Kunkel, W. 1973, ApJ, 179, 847

Knude, J., \& Høg, E. 1998, A\&A, 338, 897

Kontinen, S., Harju, J., Heikkilä, A., \& Haikala, L. K. 2000, A\&A, 361, 704

Koresko, C. D., Herbst, T. M., \& Leinert, Ch. 1997, ApJ, 480, 741

Koyama, K., Hamaguchi, K., Ueno, S., Kobayashi, N., \& Feigelson, E. D. 1996, PASJ, 48, 87 
Krügel, E., Siebenmorgen, R., Zota, V., \& Chini, R. 1998, A\&A, 331, L9

Levreault, R. M. 1988, ApJS, 67, 283

Llewellyn, R., Payne, P., Sakellis, S., \& Taylor, K. N. R. 1981, MNRAS, 196, 29P

Loren, R. B. 1979, ApJ, 227, 832

Marraco, H. G., \& Rydgren, A. E. 1981, AJ, 86, 62

Neuhäuser, R., Walter, F. M., Covino, E., et al. 2000, A\&AS, 146, 323

Olofsson, G., Huldtgren, M., Kaas, A. A., et al. 1999, A\&A, 350, 883

Ossenkopf, V., \& Henning, T. 1994, A\&A, 291, 943

Prato, L., \& Simon, M. 1997, ApJ, 474, 455

Reichertz, L. A., Weferling, B., Esch, W., \& Kreysa, E. 2001, A\&A, 379, 735

Reipurth, B., Chini, R., Krügel. E., Kreysa, E., \& Sievers, A. 1993, A\&A, 273, 221

Reipurth, B., \& Zinnecker, H. 1993, A\&A, 278, 81

Rossano, G. S. 1978, AJ, 83, 234

Saraceno, P., André, P., Ceccarelli, C., Griffin, M., \& Molinari, S. 1996, A\&A, 309, 827
SIMBA Observer's Handbook 2002, version 1.8 at http://www.ls.eso.org/lasilla/Telescopes/SEST/ html/ telescope-instruments/simba/index.html

Strom, K. M., Strom, S. E., \& Grasdalen, G. L. 1974, ApJ, 187, 83

Strom, K. M., Strom, S. E., Wolff, S. C., Morgan, J., \& Wenz, M. 1986, ApJS, 62, 39

Suters, M., Stewart, R. T., Brown, A., \& Zealey, W. 1996, AJ, 111, 320

Taylor, K. N. R., \& Storey, J. W. V. 1984, MNRAS, 209, 5P

Vaz, L. P. R. 2001, in The Formation of Binary Stars, ed. H. Zinnecker, \& R. D. Mathieu, ASP, IAU Symp., 200, 449

Wilking, B. A., Greene, T. P., Lada, C. J., Meyer, M. R., \& Young, E. T. 1992, ApJ, 397, 520

Wilking, B. A., Greene, T. P., Lada, C. J., Meyer, M. R., \& Young, E. T. 1985, ApJ, 293, 165

Wilking, B. A., McCaughrean, M. J., Burton, M. G., et al. 1997, AJ, 114, 2029

Wilking, B. A., Taylor, K. N. R., \& Storey, J. W. V. 1986, AJ, 92, 103 Yonekura, Y., Mizuno, N., Saito, H., et al. 1999, PAS Japan, 51, 911 
R. Chini et al.: SIMBA observations of the R Corona Australis molecular cloud, Online Material $p 1$

\section{Online Material}




\section{Appendix A: Data reduction}

\section{A.1. The general concept}

For the reduction of the SIMBA data, we have used the package MOPSI (Mapping On-Off Pointing Skydip Infrared). However, independent of the software that is used for the data reduction, certain steps have to be taken on the way to the final map. An extensive description is given in the SIMBA Observer's Handbook (2002).

\section{A.1.1. Conversion of the fits files}

So far, none of the available software can process the raw SIMBA fits files. Thus, these files have been converted with the simbaread software, written by R. Lemke and M. Albrecht.

\section{A.1.2. Calibration}

As primary calibrators we used the planets Uranus and Mars, since their flux densities can easilly be calculated. This step limits the accuracy of the final results significantly. The standard deviation for the conversion factor was $15 \%$.

\section{A.1.3. Baseline fitting}

The raw data contain non-astronomical emission which have to be subtracted prior to the proper data reduction. The main sources of this emission are:

- longterm fluctuations due to instabilities in the electronics;

- zero point offsets between the bolometer channels;

- spill over emission received by each bolometer channel.

Because the contribution of the non-astronomical emission changes slowly with time, it can be removed by subtracting a polynomial fit of low order (usually $<3$ ) to the data.

\section{A.1.4. Despiking}

Due to cosmic rays and electronic discharges, the raw maps contain spikes. They produce an artificially high noise level, modify the actually measured intensity or even mimic real emission. Depending on the strength of the actual source, the spikes were eliminated by cutting off signals above a certain noise level.

After despiking the baseline corrections have to be repeated.

\section{A.1.5. Deconvolution}

When using the fastscanning mode for a bolometer, one will eventually end up with a signal distribution containing negative dips. This is due to the electronics of the pre-amplifiers of the bolometer array which behave like an electric oscillator. Since the electronical design and the values of the time constants were known and stored in accessible files, a matching band-pass filter function was derived. The division of the Fourier-transformed signal by this function and the subsequent

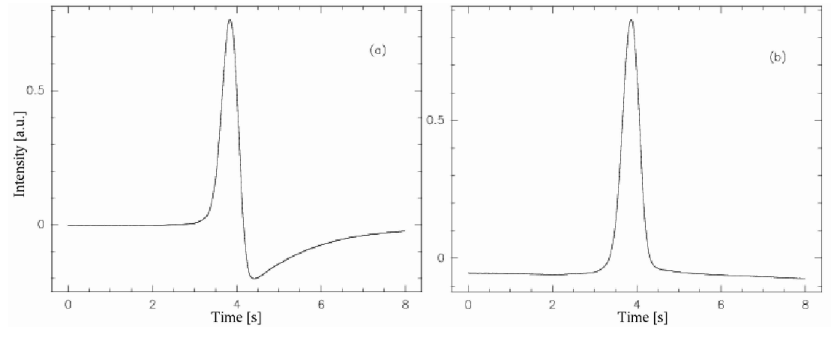

Fig. A.1. a) In the fastscanning mode, the AC-coupling of the bolometer channels causes a modification of the incoming signal. b) The application of the proper filter function restores the actual source signal (Reichertz et al. 2001).

retransformation deconvolved the signal as shown for the simplest case - the point source - in Fig. A.1.

The deconvolution algorithm for MOPSI was written by M. Anciaux, R. Lemke and M. Nielbock. It exclusively takes into account the time behaviour of the system and does not assume any particular source distribution.

\section{A.1.6. Opacity correction}

In order to obtain the zenith opacity, frequent skydips were done during the observation runs. The unextincted flux density was calculated by assuming a black body behaviour of the sky, i.e. $S_{\mathrm{em}}=S_{\mathrm{abs}}$, and simply solving the following equation for $S_{0}$ :

$S_{\mathrm{em}}=S_{0} \exp \left(\frac{-\tau}{\sin h}\right)$.

Here, $S_{\text {em }}$ stands for the measured flux density, $S_{0}$ for the unextincted flux density, $\tau$ for the zenith opacity, $h$ for the elevation and $(\sin h)^{-1}$ for the airmass. The zenith opacity $\tau$ was derived by fitting Eq. (A.1) to the skydip data.

\section{A.1.7. Skynoise reduction}

The largest contribution to the noise in the data are fluctuations in the atmosphere. The size scales of these fluctuations make sure that most of the bolometer channels experience similar changes in intensity. By eliminating the highly-correlated noise, the signal-to-noise ratio of the source was significantly increased. It therefore represented the most crucial step within the data reduction.

Ideally, a perfect skynoise reduction could be achieved if:

- the relative gains of the bolometer channels (i.e. the flat field) were correctly determined;

- the receiver system was perfectly stable;

- the system did not show any non-correlated noise;

- the fluctuations of the atmospherical emission were identical in every bolometer channel.

Since this does not represent a realistic situation, we were not able to remove the correlated noise completely. However, the comparison of data not treated with the skynoise filter as depicted in Fig. A. 2 with the final map in Fig. 1 clearly shows the difference. 


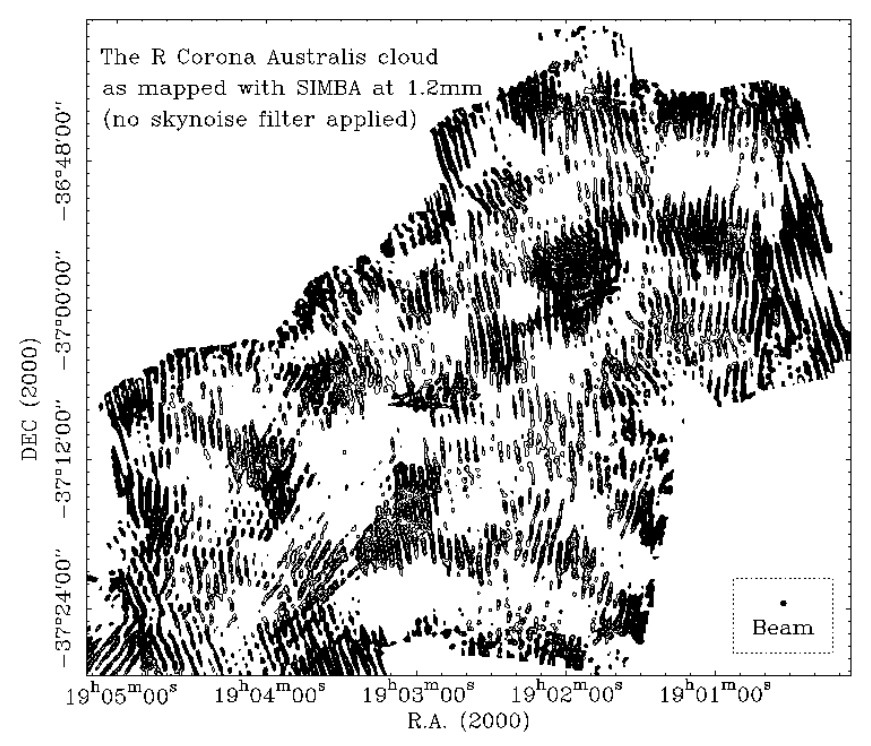

Fig. A.2. The complete $1.2 \mathrm{~mm}$ mosaic of the $\mathrm{R} \mathrm{Cr} \mathrm{A}$ dark cloud as depicted in Fig. 1. The same reduction sequence was used but no skynoise filter was applied here. The resulting mosaic suffers from strong residual noise and the distortion of the sources.

\section{A.1.8. Map creation}

In the last step, all the maps of each bolometer channel were coadded to create a final map. This step also involved a coordinate transformation from the horizontal into the equatorial system as well as the application of different weighting factors for each bolometer channel. These factors were calculated as follows:

weighting factor $\propto \frac{1}{\mathrm{rms}^{2}}$.

The rms in data of each bolometer channel was determined after the skynoise reduction and the final baseline subtraction outside the polygon defining the source.

\section{A.2. The iterative data reduction}

The following sections focus on the advanced data reduction which was performed in four iterative steps. While the basic reduction scheme was the same in each step, the settings for the skynoise filter and the baseline fitting were changed in order to improve the results.

\section{A.2.1. The first iteration}

In this first step, the data were reduced in a very basic way which, however, already included all the relevant steps as described in Appendix A.1. Since no relevant pieces of information of the possible sources were at hand, the softest settings had to be used for the reduction parameters, e.g. baselines of order 0 or 1 . On the one hand, the resulting map suffered from baseline artefacts close to the sources and strong residual noise, e.g. scanning artefacts. On the other hand, the chances that any real sources were destroyed during this first iteration were reduced to a minimum.

\section{A.2.2. The second iteration}

The second step involved the definition of a proper base range. A polygon matching the source geometry was drawn within the final map of the first iteration. This polygon was used to define a base range, i.e. the area in which the baselines are calculated. The result of the reduction showed improvements, e.g. the diminution of baseline artefacts. Furthermore, the sources had a much better signal-to-noise ratio. Nevertheless, the source structure was still not perfect and still showed residual scanning artefacts.

\section{A.2.3. The third iteration}

The results of the second iteration reduction were good enough for the creation of a source model. Because of the complicated source structure and the large amount of strong sources, the preparation of a source model (see Sect. A.2.3) demanded a lot of care. At first, two-dimensional Gaussian functions were fitted to the strongest sources (MMS 1, MMS 9, MMS 13, MMS 23 and MMS 24) and then subtracted from the mosaic. This step allowed to smooth the remaining mosaic to $40^{\prime \prime}$ without affecting the compact sources. Afterwards, the HPBW was set back to $24^{\prime \prime}$ and the formerly subtracted Gaussian fits were added. As any of the sources in the remaining mosaic is more extended than $40^{\prime \prime}$, the Gaussian smoothing did not affect the source structure. Then, a polygon was drawn around the regions of emission and the data outside this polygon was neglected. The resulting source model was used as an input for the skynoise filter of this iteration of data reduction.

At this point, further parameters of the skynoise filter could be changed. The most crucial part was still the choice of the polygon. The best results were achieved by combining an extended source model (i.e. the polygon drawn during the preparation of the model only roughly followed the morphology of the regions of emission) and the smallest possible polygon (i.e. this polygon followed the morphology of the emission very tightly). Furthermore, baselines of order three or higher were applied before, within and also after the skynoise reduction. However, the subtraction of such strong baselines did not have any significant effects on data that were taken in good or superior weather conditions and were therefore reduced to a minimum. For noisy data, the order of the baselines that could be applied without destroying sources, crucially depended on the size of the base range in comparison to the size of the map. As to now, there is no standard recipe for baselines and their order.

The result of this third iterative step showed major improvements with regard to the source structure and the quality of the baseline corrections. Slight scanning artefacts were caused by fluctuations in the electronics and therefore could not be eliminated.

\section{A.2.4. The fourth iteration}

This last step basically involved the repetition of the third iteration with further improved source model, since the results of the third iteration data reduction were usually by far better than the results of the second iteration. 


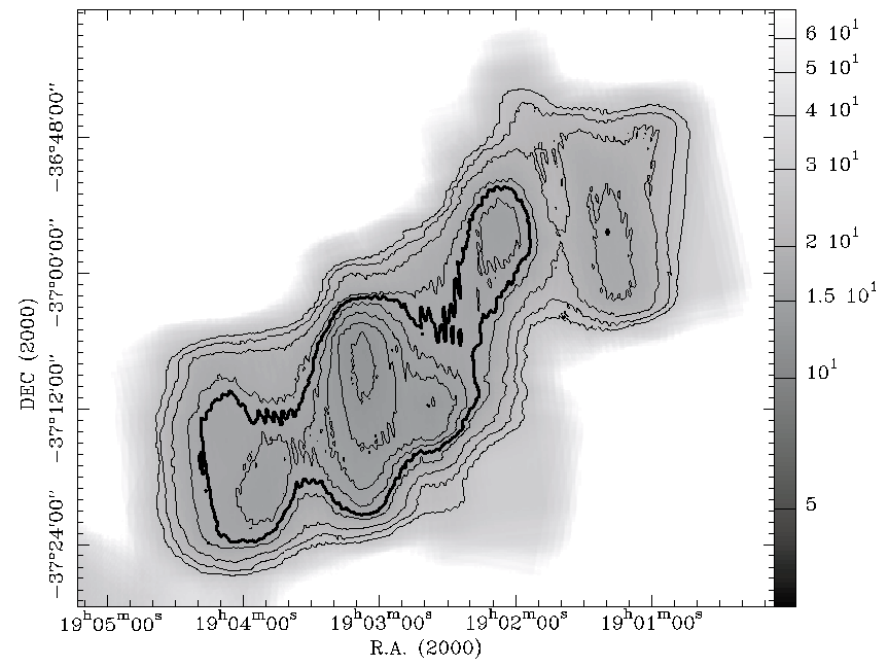

Fig. B.1. The rms-distribution of the complete $1.2 \mathrm{~mm}$ mosaic of the $\mathrm{R} \mathrm{Cr}$ A molecular cloud. The contour levels are 13 to 17 in steps of 1 , and 18 to 27 in steps of 3 . The units are in mJy/beam. The thick contour represents an rms of $17 \mathrm{mJy} /$ beam which corresponds to the assumed global rms value.

\section{Appendix B: Data analysis}

\section{B.1. Definition of the $S / N$ ratio}

The average noise in the final mosaic was determined by drawing a polygon in such a way that regions of obvious emission and the extremely noisy edges (regions with low coverages) were omitted; the rms value of fluctuations inside the polygon was defined as the noise which determines the detection limit of the faintest sources. The validity of this procedure was tested by calculating the rms within smaller polygons across the entire mosaic; the variations found in this way turned out to be $\pm 2 \mathrm{mJy} /$ beam. Due to the complex morphology of dust emission in $\mathrm{R} \mathrm{Cr} \mathrm{A}$, it is almost impossible to distinguish between the emission from a compact source and the emission originating from a diffuse dust continuum. Likewise, it was not possible to determine the local $\mathrm{rms}$ on certain high emission plateaus. Consequently, the $S$ in the $S / N$ ratio stands for the total signal measured at the position of the peak whereas the $N$ represents the global rms noise of the background.

\section{B.2. The rms distribution}

The values of the rms of the final mosaic depend on the rms of each single bolometer channel and the sampling in the equatorial system (note: maps are originally taken in the horizontal system). Figure B.1 depicts the rms distribution over the entire mosaic. 